\title{
Medical Image Segmentation of Improved Genetic Algorithm Research Based on Dictionary Learning
}

\author{
Xianqi Cao1, Jiaqing Miao',3, Yu Xiao ${ }^{3}$ \\ ${ }^{1}$ College of Applied Technology, University of Science and Technology Liaoning, Anshan, China \\ ${ }^{2}$ School of Mathematical Sciences, University of Electronic Science and Technology of China, Chengdu, China \\ ${ }^{3}$ Engineering and Technical College, Chengdu University of Technology, Leshan, China \\ Email: caoxq905@163.com
}

How to cite this paper: Cao, X.Q., Miao, J.Q. and Xiao, Y. (2017) Medical Image Segmentation of Improved Genetic Algorithm Research Based on Dictionary Learning. World Journal of Engineering and Technology, 5, 90-96.

https://doi.org/10.4236/wjet.2017.51008

Received: January 18, 2017

Accepted: February 21, 2017

Published: February 24, 2017

Copyright $\odot 2017$ by authors and Scientific Research Publishing Inc. This work is licensed under the Creative Commons Attribution International License (CC BY 4.0).

http://creativecommons.org/licenses/by/4.0/

\begin{abstract}
The image signal is represented by using the atomic of image signal to train an over complete dictionary and is described as sparse linear combinations of these atoms. Recently, the dictionary algorithm for image signal tracking and decomposition is mainly adopted as the focus of research. An alternate iterative algorithm of sparse encoding, sample dictionary and dictionary based on atomic update process is K-SVD decomposition. A new segmentation algorithm of brain MRI image, which uses the noise reduction method with adaptive dictionary based on genetic algorithm, is presented in this paper, and the experimental results show that the algorithm in brain MRI image segmentation has fast calculation speed and the advantage of accurate segmentation. In a very complicated situation, the results show that the segmentation of brain MRI images can be accomplished successfully by using this algorithm, and it achieves the ideal effect and has good accuracy.
\end{abstract}

\section{Keywords}

Dictionary, K-SVD, Matching Pursuit, Sparse Representation, Genetic Algorithm, Dual Population

\section{Introduction}

The technology of brain MRI image segmentation is the key of clinical diagnosis of brain medicine, and brain magnetic resonance image segmentation is an important step in medical diagnosis, which can not only be used to estimate and detect the lesion area, but also is an important basis for doctors to carry out the operation and make the diagnosis plan. So far, the main methods of brain MRI 
image segmentation are mainly based on classification method, filtering method and so on, and there are some disadvantages of the corresponding segmentation regions, for example, the inaccuracy of narrow focus area segmentation, sensitive to the image noise and other issues. Especially in the case of complex problems, some algorithms are simply not working. This paper presents a new segmentation algorithm of brain magnetic resonance imaging, using the method of combining adaptive dictionary and noise reduction based on genetic algorithm. The genetic algorithm does not require describing all of the features of the problems beforehand, and can also result in robust solutions to complex problems. But it has many disadvantages, because of these shortcomings, which lead to the deviation in medical image segmentation, and even wrong segmentation.

In this paper, on the basis of the traditional population, it adds a sub population, so that the two populations can result in more individuals and overcome the instability through competition. Numerical experiments show that the algorithm proposed in the brain MRI medical image segmentation application has fast calculation speed and accurate segmentation characteristics. The results of synthetic image segmentation show that the proposed algorithm which is better than the traditional genetic algorithm, has better robustness and can eliminate the interference of noise. When the noise pollution is more serious, the segmentation advantage of this algorithm can be better reflected.

\section{K-SVD Dictionary Algorithm for Brain MRI Medical Image}

\subsection{K-SVD Algorithm Description}

$\mathrm{K}-\mathrm{SVD}$ is given a set of signals in strict constraints under the conditions of the actual training [1]. This paper shows the results of image restoration on the real medical images. Specific algorithms are as follows:

1) Purpose: Find an optimal dictionary sparse representation $\left\{y_{i}\right\}_{i=1}^{N}$ by solving the following equation.

$$
\min _{D, X}\left\{\|Y-D X\|_{F}^{2}\right\} \text { s.t. } \forall i,\left\|X_{i}\right\|_{0} \leq T_{0} .
$$

2) Initialization: Create a dictionary $D^{0} \in R^{n \times K}$. Assume $J=1$.

3) Repeat the following steps until the stop condition is met:

a) Sparse coding: Calculate the following formula $x_{i}$, the expression vector of $y_{i}$, by the tracking algorithm

$$
\min _{x_{i}}\left\{\left\|y_{i}-D x_{i}\right\|_{2}^{2}\right\} \text { s.t. }\left\|x_{i}\right\|_{0} \leq T_{0}, \quad i=1,2, \cdots, N
$$

b) Password update procedure: Each column of $D^{(j-1)}$ update through the following steps:

1) Define a set of atomic samples $\omega_{k}$.

2) Calculate all error matrixes $E_{k}$ :

$$
E_{k}=Y-\sum_{j \neq k} d_{j} x_{T}^{j}
$$

3) Limit $E_{k}^{R}$ by selecting the same column as $E_{k}$, and obtain $E_{k}^{R}$. 
4) Decompose $E_{k}^{R}$ by singular value, and obtain $E_{k}^{R}=U \Delta V^{T}$, Choose $U$ 's first column as a list of $d_{k}$.

5) $J=J+1$.

The algorithm uses SVD to update the coefficients of the corresponding algorithm [2]. In addition, each step of $d_{k}$ 's update process is decreasing, so as to ensure convergence to the local minimum

\subsection{Results and Analysis of Brain MRI Medical Image Denoising}

In Figure 1, an adaptive K-SVD learning dictionary for MRI and CT medical images is given, in which the size of a small piece of medical image is $8 \times 8$. Among them, Figure 1(a) for MIR and CT image, Figure 1(b) for K-SVD dictionary training adaptive learning, Figure $1(c)$ is a denoising result using K-SVD learning dictionary.

The results show that the adaptive dictionary denoising is an effective dictionary learning algorithm [3]. In this paper, a complete dictionary learning algorithm is used to denoise the brain MRI medical image, and the improved dual population genetic algorithm is adopted to segment the medical image.

\section{Improved Dual Population Genetic Algorithm for Dictionary Learning}

\subsection{Principle of Image Segmentation Based on Dual Population Genetic Algorithm}

The evolutionary process of nature is the process of continuous iteration of the basic factors of reproduction, selection, crossover and mutation, so it can be regarded as an optimization problem to solve the optimal value of the process [4]. The double population genetic algorithm is mainly based on the idea of competition. If it wants to evolve in a better direction, it needs to compete, but also to exchange information among populations.
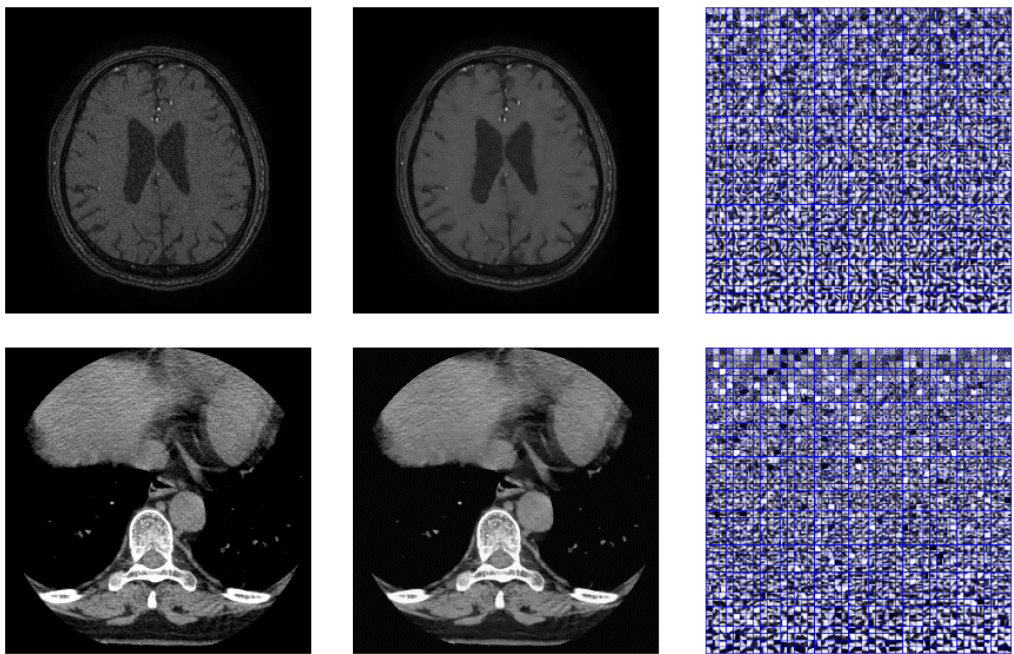

(a)

(b)

(c)

Figure 1. Global dictionary learning denoising image. 
Two different individuals $p_{1}$ and $p_{2}$, in which the same genes account for all of the genes is called similarity. That is, their similarity is $S\left(P_{1}, P_{2}\right)=m / n$, among them, $n$ is the total number of genes [5] [6], $m$ is the number of the same gene in two individuals.

The similarity between all individuals is found by using Hamming distance. Hamming distance is defined as follows:

$$
H_{i, j}=\sum_{k=1}^{n}\left|a_{i k}-a_{j k}\right|
$$

where $n$ is the total number of genes, $a_{i k}$ is the binary of $k$ gene in $i$ individual.

In the dual population genetic algorithm, it is usually to introduce good individuals and eliminate the bad individuals. In this paper, the fitness of individuals in the population is arranged from large to small, and the competition among populations is accomplished by similarity comparison.

Through the calculation of Hamming distance, similar individuals will cross with other populations, then compute the fitness of function and select the dominant population. At the same time, in order to make the diversity of the form of the population, the sub population will regenerate a new individual.

\subsection{Medical Image Segmentation Based on Improved Dual Population Genetic Algorithm}

The improvement of double population genetic algorithm realizes the segmentation of medical image [7], and the fitness function is expressed as follows:

$$
\sigma_{s}(s, t)=\frac{S_{b}(s, t)}{S_{w}(s, t)}
$$

and:

$$
\begin{aligned}
S_{b}(s, t)= & \sum_{i=1}^{s} \sum_{j=1}^{t} w_{b}\left(\left|i-\mu_{l 1}\right|+\left|j-\mu_{l 1}\right|\right) \\
& +\sum_{i=s+1}^{L} \sum_{j=t+1}^{L} w_{o}\left(\left|i-\mu_{l 2}\right|+\left|j-\mu_{l 2}\right|\right) \\
S_{w}(s, t)= & w_{b}(s, t) d_{b}(s, t)+w_{o}(s, t) d_{o}(s, t)
\end{aligned}
$$

\subsection{Experimental Results and Analysis of Dictionary Learning Improved Genetic Algorithm}

All the models are tested by MATLAB R2014a software in the Intel CPU Core i5-4590@3.30 GHz, 8 GB of memory on the computer. Parameters of genetic algorithm: population has 30 individuals, mutation probability $p_{m 1}$ is 0.005 and $p_{m 2}$ is 0.001 , and evolutionary genetic algebra is 300 . The improved algorithm and the dictionary learning algorithm are combined to realize the medical MRI image segmentation, and each of segmentation is carried out 10 independent experiments to get the average value. The experimental results are shown in Figure 2.

From the visual point of view, in the results of brain MRI medical image 

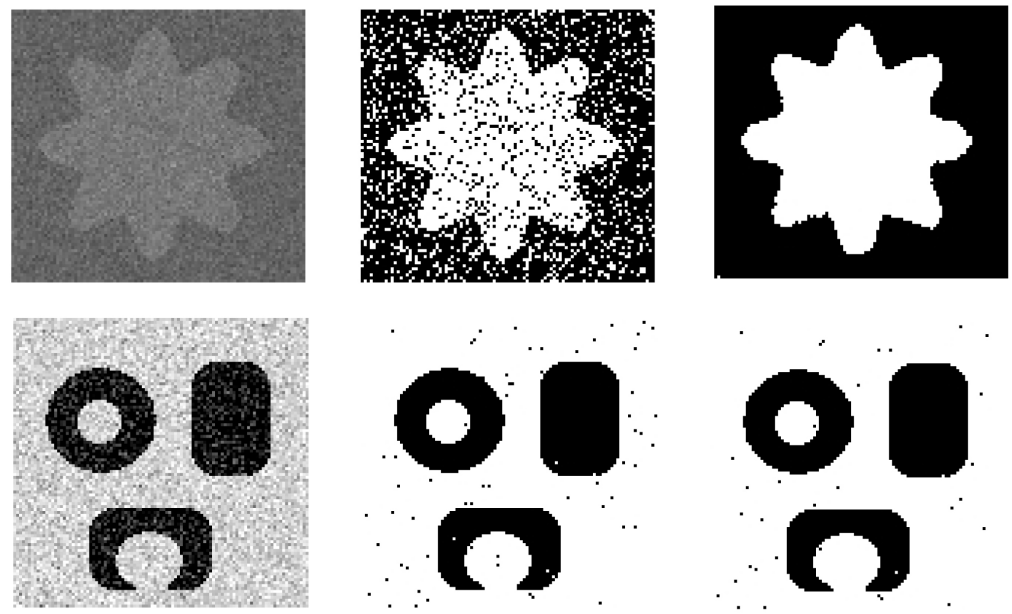

(a)

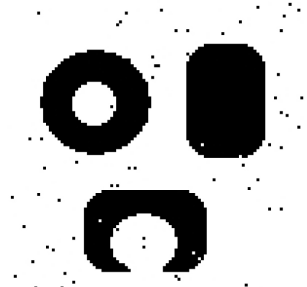

(b)

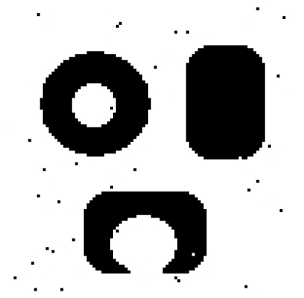

(c)

Figure 2. Synthetic image segmentation based on Improved Genetic Algorithm. (a) Original composite image; (b) Dual population genetic algorithm; (c) Dictionary learning improved genetic algorithm.

segmentation, the improved algorithm based on dictionary learning [8] [9] is more effective and accurate than double population genetic algorithm segmentation.

\subsection{Performance Assessment}

In the experiment, the initial population contains 40 individuals and the crossover rate is changing. Mutation probability $p_{m 1}$ is $0.005, p_{m 2}$ is 0.005 , and max evolutionary genetic algebra is 500. From the results of the segmentation from Figures 2-5, it can be seen that the improved double population genetic algorithm can find the optimal solution by tenth generations. The improved genetic algorithm based on dictionary learning, which can be used to separate multiple targets and control the noise better than dual population genetic algorithm, can better reflect the advantages of segmentation when the noise pollution is more serious. Thus, the combination of genetic algorithm based on dictionary learning and improved genetic algorithm can be improved MRI medical image segmentation accuracy in MRI image segmentation.

\section{Conclusion}

An improved dual population genetic algorithm, which is proposed to solve the problem of double population genetic algorithm in the paper, is stable and overcomes the premature phenomenon by experimental results; however, it is not robust to noise. In the brain MRI medical image, the segmentation results, which are produced by a noise reduction method based on adaptive dictionary learning that is introduced in the dual population improved genetic algorithm, show that the proposed algorithm has higher stability, more accurate segmentation, but also greatly reduces the computational complexity of genetic algorithm. Through the study of the brain MRI image segmentation results, this algorithm 

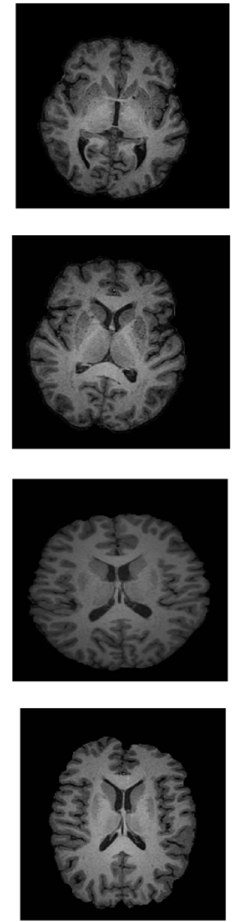

(a)

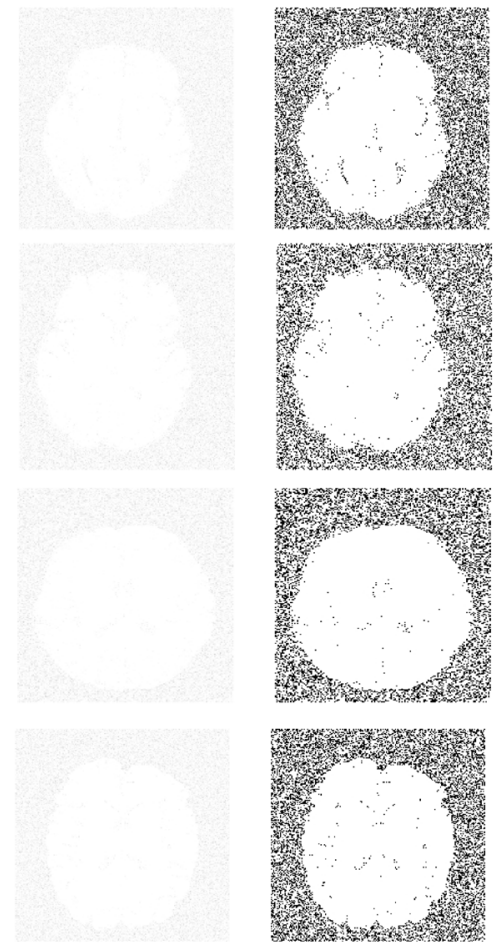

(b)
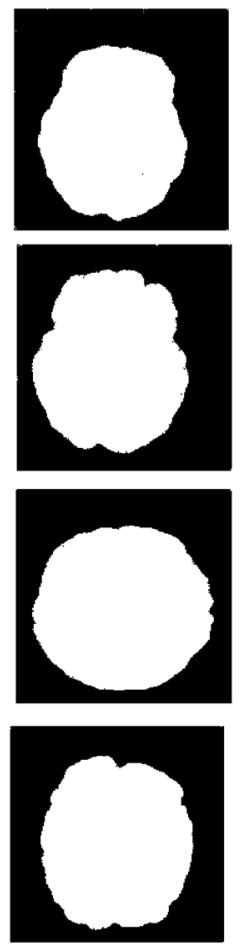

(d)

Figure 3. Low contrasts MRI image segmentation based on improved dictionary learning genetic algorithm. (a) Low contrast learning MRI image; (b) MRI image after Gauss noise pollution; (c) Improved dual population genetic algorithm; (d) Dictionary learning dual population genetic algorithm.
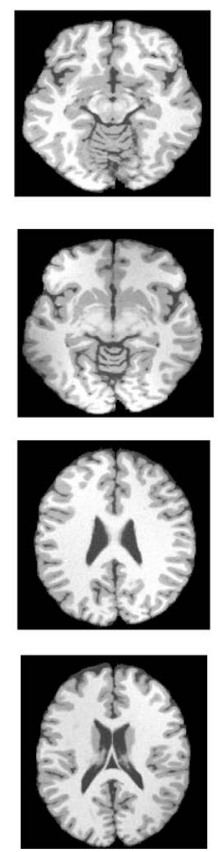

(a)
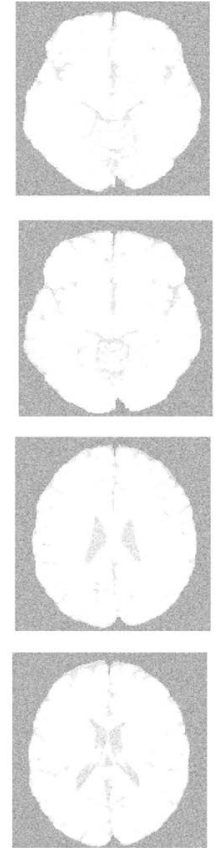

(b)
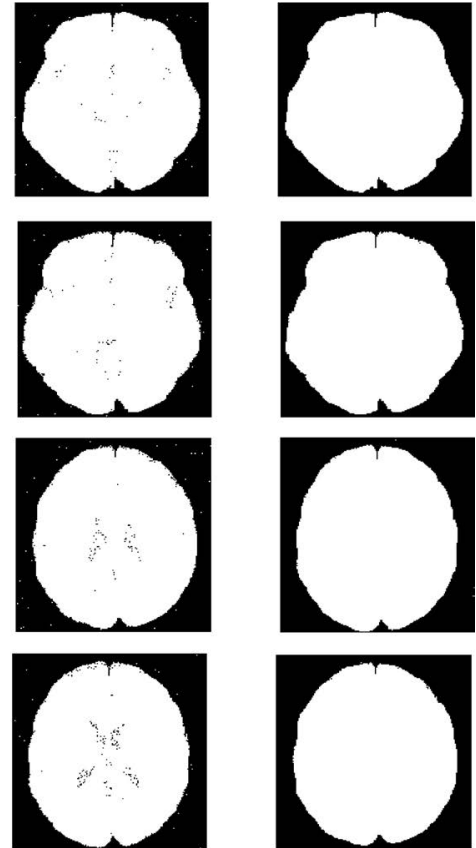

(c)
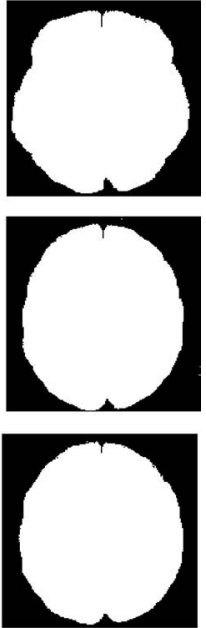

(d)

Figure 4. High contrasts MRI image segmentation based on improved dictionary learning genetic algorithm. (a) High contrast learning MRI image; (b) MRI image after Gauss noise pollution; (c) Improved dual population genetic algorithm; (d) Dictionary learning dual population genetic algorithm. 

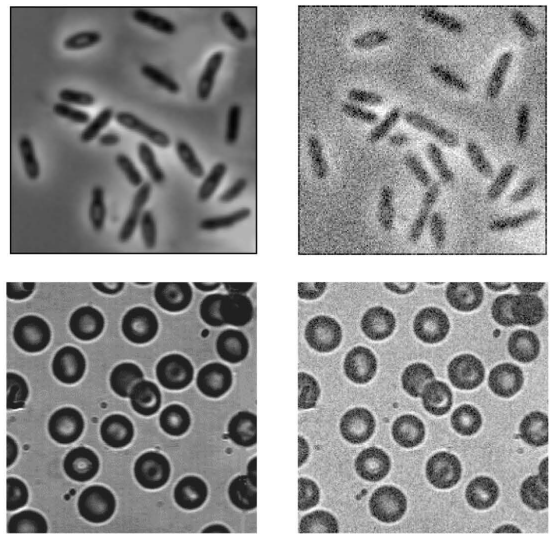

(a)

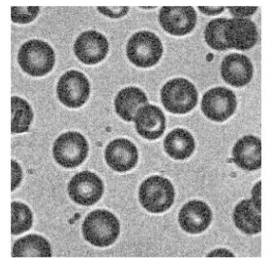

(b)
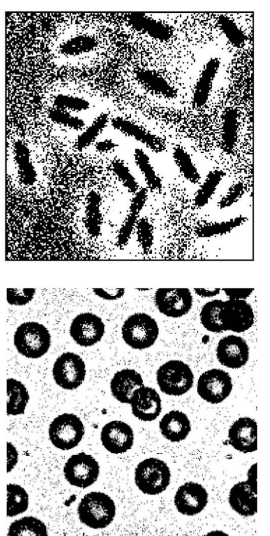

(c)
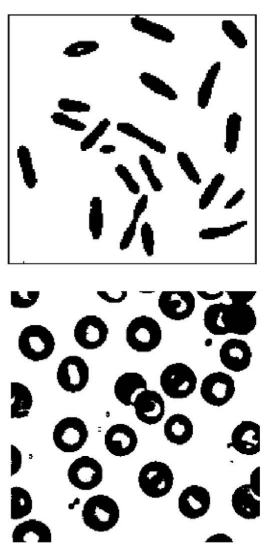

(b)

Figure 5. Bacterial image segmentation based on improved genetic algorithm based on dictionary learning. (a) Original bacterial image; (b) Bacterial image after Gauss noise pollution; Improved dual population genetic algorithm; (d) Dictionary learning dual population genetic algorithm.

has better portability in practical applications, and is more easy to popularize.

\section{References}

[1] Cai, Z. and Lai, J. (2009) An Over-Complete Learned Dictionary-Based Image De-Noising Method. Acta Electronica Sinica, 37, 347-350.

[2] Jiang, Z., Lin, Z. and Davis, L.S. (2013) Label Consistent K-SVD: Learning a Discriminative Dictionary for Recognition. IEEE Transactions on Pattern Analysis and Machine Intelligence, 35, 2651-2664. https://doi.org/10.1109/TPAMI.2013.88

[3] Yang, M. and Zhang, G. (2012) SAR Images De-Speckling Algorithm via an Adaptive Over-Complete Learning Dictionary. Journal of Image and Graphics, 4, 596602.

[4] Deb, K., Pratap, A., Agarwal, S., et al. (2002) A Fast and Elitist Multiobjective Genetic Algorithm: NSGA-II. IEEE Transactions on Evolutionary Computation, 6, 182-197. https://doi.org/10.1109/4235.996017

[5] Pezzella, F., Morganti, G. and Ciaschetti, G. (2008) A Genetic Algorithm for the Flexible Job-Shop Scheduling Problem. Computers \& Operations Research, 35, 32023212. https://doi.org/10.1016/j.cor.2007.02.014

[6] Whitley, D. (2014) An Executable Model of a Simple Genetic Algorithm. Foundations of Genetic Algorithms, 2, 45-62.

[7] Çam, E., Lüy, M. and Mamur, H. (2016) PID Parameter Optimization of Blade Pitch Controller in Wind Turbines by a New Intelligent Genetic Algorithm. IET Renewable Power Generation, 10, 1220-1228.

[8] Zwickl, D.J. (2006) Genetic Algorithm Approaches for the Phylogenetic Analysis of Large Biological Sequence Datasets under the Maximum Likelihood Criterion. The University of Arizona, Tucson.

[9] Grefenstette, J.J. (2013) Genetic Algorithms and Their Applications: Proceedings of the Second International Conference on Genetic Algorithms. Psychology Press, Hove. 
Submit or recommend next manuscript to SCIRP and we will provide best service for you:

Accepting pre-submission inquiries through Email, Facebook, LinkedIn, Twitter, etc. A wide selection of journals (inclusive of 9 subjects, more than 200 journals)

Providing 24-hour high-quality service

User-friendly online submission system

Fair and swift peer-review system

Efficient typesetting and proofreading procedure

Display of the result of downloads and visits, as well as the number of cited articles Maximum dissemination of your research work

Submit your manuscript at: http://papersubmission.scirp.org/

Or contact wjet@scirp.org 